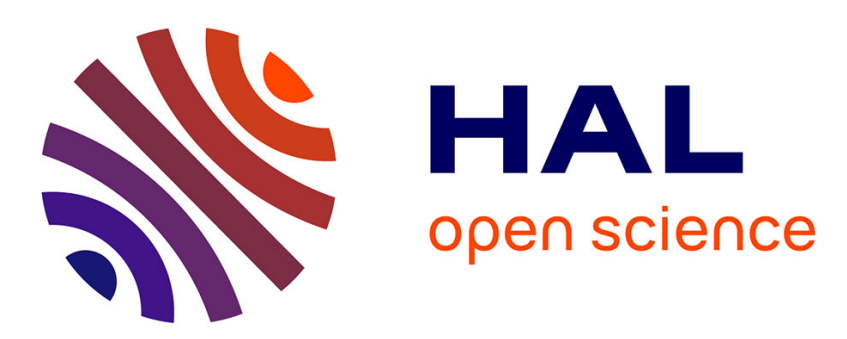

\title{
A layerwise finite element for multilayers with imperfect interfaces
}

van Anh Duong, Alberto Diaz Diaz, Sylvain Chataigner, Jean-François Caron

\section{To cite this version:}

van Anh Duong, Alberto Diaz Diaz, Sylvain Chataigner, Jean-François Caron. A layerwise finite element for multilayers with imperfect interfaces. Composite Structures, 2011, 93 (12), pp.3262-3271. 10.1016/j.compstruct.2011.05.001 . hal-00639548

\section{HAL Id: hal-00639548 https://hal.science/hal-00639548}

Submitted on 16 Mar 2018

HAL is a multi-disciplinary open access archive for the deposit and dissemination of scientific research documents, whether they are published or not. The documents may come from teaching and research institutions in France or abroad, or from public or private research centers.
L'archive ouverte pluridisciplinaire $\mathbf{H A L}$, est destinée au dépôt et à la diffusion de documents scientifiques de niveau recherche, publiés ou non, émanant des établissements d'enseignement et de recherche français ou étrangers, des laboratoires publics ou privés. 


\title{
A layerwise finite element for multilayers with imperfect interfaces
}

\author{
Van Anh Duong a ${ }^{\text {, Alberto Diaz Diaz }}{ }^{\mathrm{b}}$, Sylvain Chataigner a , Jean-François Caron ${ }^{\mathrm{a}, *}$ \\ ${ }^{a}$ Université Paris-Est, Institut Navier, Ecole des Ponts, 6-8 Avenue Blaise Pascal, 77455 Marne La Valle Cedex 2, France \\ ${ }^{\mathrm{b}}$ Centro de Investigación en Materiales Avanzados (CIMAV), Miguel de Cervantes 120, Complejo Industrial Chihuahua, 31109 Chihuahua, Mexico
}

\begin{abstract}
This article is aimed at proposing a new development of a layer-wise 2D finite-element method for multilayers considering the laminated plate as a superposition of Reissner plates coupled by interfacial stresses. Here, despite the 2D description of the laminates, the interfaces show a particular behavior, elastic or elastoplastic (Von-Mises criterion). The finite element formulation is derived and an eight-node multiparticle element is detailed. The application example of a double lap joint with an elastoplastic adhesive is then considered. The adhesive layer is modeled as an interface. The loading scheme is a load-unload-load one. Interface shear and normal stresses are compared to 3D finite element results. A good agreement between both techniques is observed, particularly for the prediction of the history of the slip between the two adherends and the plastic strains in the adhesive.
\end{abstract}

\section{Introduction}

In order to answer to an increasing demand of multifunctionality and optimization, classical structures are becoming more and more complex, including multilayered parts instead of monolithic solutions, and specific interfaces as bonding or connectors. These designs also need complex numerical tools able to predict the mechanical behavior with accuracy. For laminated structures, it is well known that high interlaminar stresses play a significant role not only in local but also in global failure mechanisms. Therefore, a correct modelling of interfaces is required.

Interfaces are two-dimensional entities between two layers in which the stress vector continuity is ensured. Usually, a perfect interface is considered and the 3D displacement field is then continuous across it. Very thin layers may be modelled as perfect interfaces in order to simplify the analysis. This is the case, for example, of thin layers of an adhesive material exhibiting small strains in an adhesively bonded joint. But, in this example, when large strains in the thin adhesive layer occur, the discontinuity of displacements across the interface may be non-negligible (the interface is imperfect). In [1], by testing composite laminates made up of carbon-epoxy prepregs, Diaz and Caron measured displacement discontinuities (across interfaces) as large as one tenth of the thickness of the plies before debonding of layers (Fig. 1) and a delamination criterion involving a critical interlaminar sliding value was proposed. In [2], Le Roy et al. proved that a correct

\footnotetext{
* Corresponding author. Fax: +3316015 3741 .

E-mail address: caron@enpc.fr (J.-F. Caron).
}

modeling of concrete-wood composite beams with discrete connectors requires the consideration of imperfect interfaces, too.

Another important issue in the modeling of laminated structures is the technique for evaluating stresses. The use of a 3D approach is perhaps not the best choice; on the one hand, because tools have to be as simple and as cheap as possible to be useful to the designer. On the other hand, because the answers provided by these simulations, are too sharp, not enough global or macroscopic, and, moreover, not always relevant (mesh dependency, presence of singularities, size of heterogeneities not adequate for the level of description). Therefore, post-processing is necessarily used. For example, integrations on areas defined by the operator can provide converging values, independent of the mesh [3-5].

Due to all these difficulties, it seems more natural to calculate with $2 \mathrm{D}$ approaches the multilayer which has a plate topology. It is necessary to exhibit relevant global variables, which lie on rigorous mechanical concepts and properly describe the phenomena. This topic has been handled by the research community for several years. In the first part of this article, a review of the available 2D modelling is presented. In a second part, the authors describe their own approach, and more specifically, the possibility to introduce an elastoplastic interface sliding. Finally, and since the plasticity smoothes in this case the 3D singularities, the 2D finite element model is compared to a 3D finite element approach in the case of an elastoplastic double lap joint.

\section{2D plate model for composite materials}

A very complete review of these models can be found in [6], where continuum based models, and asymptotic or axiomatic type 


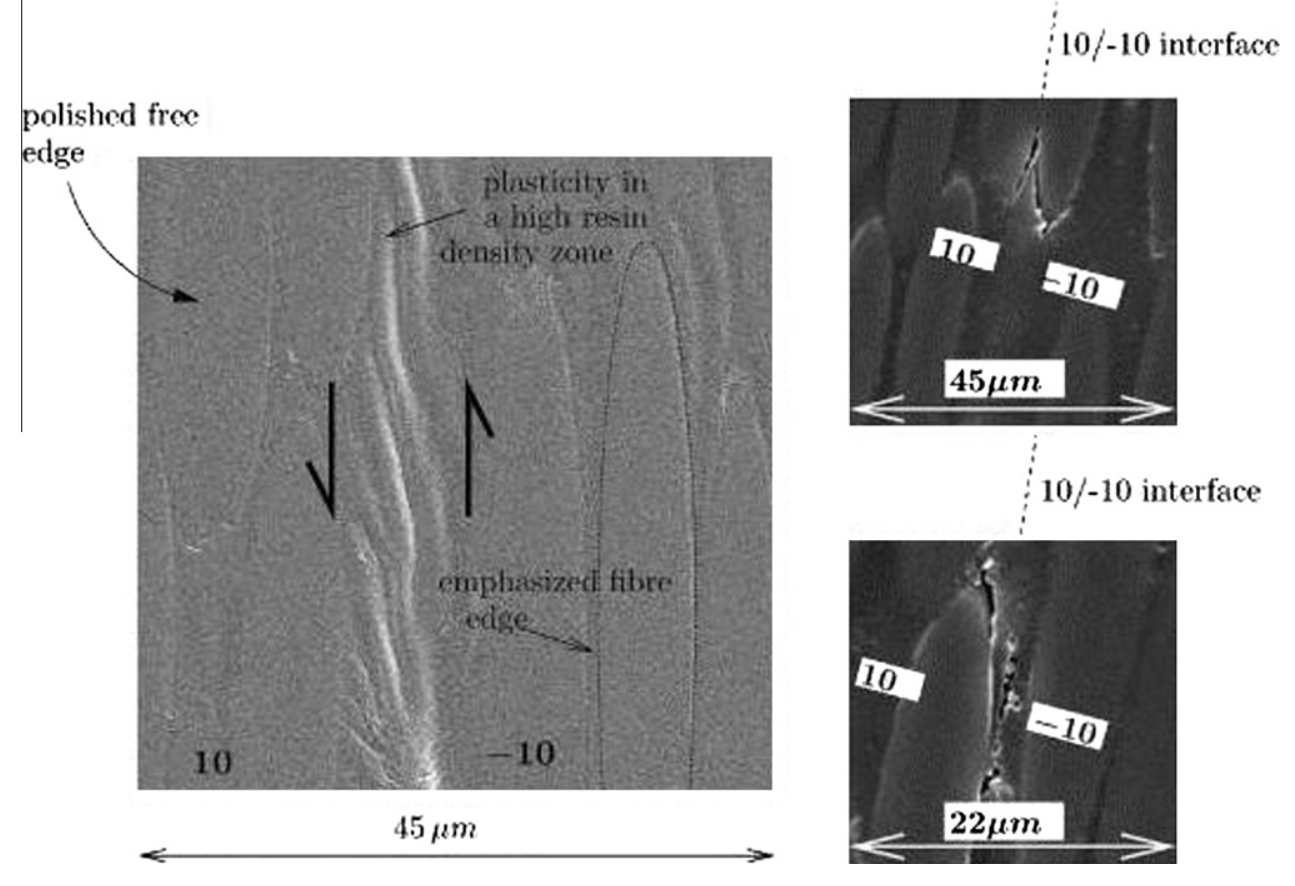

Fig. 1. Plastic sliding in a composite interface [1].

approaches are listed. Here, the same proposed classification and the same focus on the very large family that E. Carrera described as axiomatic is adopted. These theories postulate a certain intuitive displacement and/or stress field form in the thickness $z$-direction. They may also consider the multilayer as an equivalent single layer or as if each layer is seen as an independent plate [7]. In the first case, the models derived from the classical plate theories, for instance, the Love-Kirchhoff theory or the Reissner-Mindlin theory. They differ in the degree of the $z$ polynomial choices for displacement fields (rarely for stress fields), and in some other assumptions. For example, the Classical Lamination Theory $[7,8]$ neglects the out-of-plane strains, when the First Order Theory $[9,10]$ includes these important effects for composite structures. Some models involve different kinematics such as Cosserat [11] but most of developments concern higher order theories which provide a better description of fields by increasing the degree of polynomials in the $z$-direction (see $[6,12]$ ) or by adding local interpolation strategies during the numerical implementation (see [13]). These models are rather intended to a global design and are not designed for predicting local phenomena, as those due to free edges or interfaces. However, the operational advantage is undeniable since these approaches permit a plate description with a number of degrees of freedom independent from the number of layers. In the same way, some authors propose the socalled zig-zag models (a complete list can be found in [6] and an historical review in [14]), which keep this last advantage while improving the kinematic description, by taking into account brutal mechanical property changes across interfaces. For instance, in $[15,16]$, a piecewise linear function with a slope changing at each interface, is added to the global Equivalent Single Layer displacement. In [17], a sinus function replaces the linear piecewise function and then smoothes the changes in the displacement field. The field descriptions are finer with these zig-zag approaches (conditions of stress continuities can also be added) and they provide a good trade-off and correct solutions for thick structures, for example.

However, if the objective is to capture more specific features as interface stresses or edge effects, the natural way is to implement a so-called layer-wise description. The multilayer can then be shown as a superposition of membranes or plates, each of them having their own kinematic or stress description. An outcome of this modeling is a number of degrees of freedom which depends on the number of layers, but the displacement or stress approximation can be very close to the $3 \mathrm{D}$ solution. A review of these approaches is proposed again in [6]. The models differ in the choice of the approximated fields: displacements $[8,18]$ or displacements and stresses $[15,19]$. Pure stress approaches are less usual. However, the study of singularities seems more natural, more convenient with a better and direct description of stress fields. A noteworthy work has been made in this way by Pagano [20]. Using a Hellinger-Reissner variational mixed formulation [21] and a stress field approximation, an efficient model is derived. The key point which is not often highlighted, is that no displacement approximations are made, despite the use of a mixed formulation. 2D generalized displacements are shown, energetically associated with the generalized forces which derive from the stress approximation, but no constraint conditions on the 3D displacement fields are established. "Note that we refrain from assuming the form of the displacement field in accordance with the objectionable features of that approach" ([20] p.389). It leads to a less constrained model than those where both displacement and stresses components are approximated. A more recent development of this method can be found in $[6,22]$ where simplifications are made providing a more operational formulation. This model was being already widely validated [23]. A quadrilateral $\mathbb{C}^{0}$ finite element model (MPFEAP for Multi Particle Finite Element Analysis Modeling) deriving from this model was also developed [24]. In the next part of this paper the equations are briefly summarized, and the new developments with elastic or plastic interface sliding are detailed.

\section{A layerwise model with imperfect interfaces}

The model called $\mathcal{M} 4-5 n$, initially developed for calculating interface stresses, [25,22], is specifically devoted to the study of the interface phenomena, delamination initiation or sliding. In the initial formulation, interfaces are considered as perfect (outof-plane stresses continuity, infinitely rigid) and the applications used an estimation of interface stresses for criterion proposals $[23,1]$. But such an approach reaches its own limit very quickly, since the interfaces can be more complex. For example, discrete 
connectors in concrete-wood composite beams [2], elastoplastic bonds [26,27], honeycombs sandwich [28] or damageable interface [29] exhibit a strongly non-linear behavior.

There are several ways to manage such specificities. The 3D finite element is once again not the most adapted model, since the interface concentrates high gradients (even singularities) in a weak dimension with regard to those of the layers. It is therefore difficult to use acceptable meshing for both adherends and a thin interface. From a numerical point of view, it leads to problems with too large extensions.

Consequently, it is classical to represent joints (with weak thickness and often weak rigidity) with cohesive elements, and to develop specially interface finite elements, with no thickness, which link stresses in the element and displacements close to the interface [29]. A damage model is generally introduced to create delamination [30-34].

Taking into account the weak thickness and weak rigidity, several analytical works propose also an asymptotic study completed by numerical calculations. In these mathematical studies [35-37], the thickness tends to zero and the interface is replaced by a constraint condition between layers, whether they are linear or not [38].

In this layerwise approach, several steps were taken in this sense. Firstly, in [1] the inelastic interface was introduced as a non-elastic strain in the analytical model permitting the sliding between two layers of composite (Fig. 1). An elastoplastic behavior was then applied to the law which links the generalized interlaminar stresses to the displacement dicontinuities across the interface. This law was fitted to experimental data ([1], Fig. 1 left panel). The described interface is not yet really physical since it has not its own behavior.

For thicker and more elastoplastic interfaces, as some bonding joints ([39], Fig. 2), it might be interesting to introduce in the model a more representative behavior of such an interface.

In the following part, the description of the model and the main governing equations are briefly reminded, the interface behavior is detailed, the 2D finite element is presented, and an application on an elastoplastic double lap joint is proposed as a validation of the 2D approach.

\subsection{Description and notations}

The multilayered plate is then composed of $n$ orthotropic elastic layers bonded together.

- Each layer $i$, thickness $e^{i}$, is bounded by the lower surface $h_{i}^{-}$and the top surface $h_{i}^{+}$. The average surface is noted by $\bar{h}_{i}$. Between two adjacent layers $i$ and $i+1$, the adhesive has a thickness $e^{i, i+1}$
- The volume occupied by the plate is $\Omega=\omega \times\left[h_{1}^{-}, h_{n}^{+}\right]$

- The superscripts $i$ and $j, j+1$ indicate layer $i$ and the interface between layers $j$ and $j+1(i=1, \ldots, n$ and $j=1, \ldots, n-1)$.

- The subscripts $a$ stand for the adhesive layer.

- The Greek subscripts $\alpha, \beta, \gamma, \delta$ indicate the components on the $(x, y)$ plane and are assigned the values 1 and 2 . Subscript 3 indicates the normal direction $z$.

- Tensors, matrices and vectors are expressed in bold face characters.

\subsection{Governing equations}

The stress state of each layer $i$ is described by the following generalized resultants and stresses (details in [25]): the in-plane stress resultant $N_{\alpha \beta}^{i}$, the in-plane moment resultant $M_{\alpha \beta}^{i}$, the out-of-plane shear stress resultant $Q_{\alpha \beta}^{i}$ and the interlaminar shear and normal stresses at the interface $j, j+1, \tau_{\alpha}^{\mathbf{j} \mathbf{j}+\mathbf{1}}$ and $v^{j j^{j+1}}$.

$$
\begin{aligned}
& N_{\alpha \beta}^{i}(x, y)=\int_{h_{i}^{-}}^{h^{+}} \sigma_{\alpha \beta}(x, y, z) d z \\
& M_{\alpha \beta}^{i}(x, y)=\int_{h_{i}^{-}}^{h^{+}}\left(z-\bar{h}_{i}\right) \sigma_{\alpha \beta}(x, y, z) d z \\
& Q_{\alpha}^{i}(x, y)=\int_{h_{i}^{-}}^{h^{+}} \sigma_{\alpha 3}(x, y, z) d z \\
& \tau_{\alpha}^{j, j+1}(x, y)=\sigma_{\alpha 3}\left(x, y, h_{j}^{+}\right) \\
& v^{j . j+1}(x, y)=\sigma_{33}\left(x, y, h_{j}^{+}\right)
\end{aligned}
$$

Assuming a linear distribution of the in-plane 3D stresses through each layer thickness (and by the way, parabolic and third order transverse shear and normal stresses distribution), it can be shown [25] that the following in-plane elastic strains $\epsilon_{\alpha \beta}^{i}$, the curvature $\chi_{\alpha \beta}^{i}$ and the transverse strains $\gamma_{\alpha}^{i}$ are the generalized elastic strains associated from an energetic point of view to the generalized resultants, respectively $N_{\alpha \beta}^{i}, M_{\alpha \beta}^{i}, Q_{\alpha}^{i}$. For the same reason, the generalized interlaminar elastic displacements $D_{\alpha}^{e j, j+1}, D_{3}^{e j, j+1}$ are associated with $\tau_{\alpha}^{j, j+1}$ and $v^{j, j+1}$

$$
\begin{aligned}
& N_{\alpha \beta}^{i} \leftrightarrow \varepsilon_{\alpha \beta}^{i}=\frac{1}{2}\left(U_{\alpha, \beta}^{i}+U_{\beta, \alpha}^{i}\right) \\
& M_{\alpha \beta}^{i} \leftrightarrow \chi_{\alpha \beta}^{i}=\frac{1}{2}\left(\phi_{\alpha, \beta}^{i}+\phi_{\beta, \alpha}^{i}\right) \\
& Q_{\alpha}^{i} \leftrightarrow \gamma_{\alpha}^{i}=\phi_{\alpha}^{i}+U_{3, \alpha}^{i} \\
& \tau_{\alpha}^{j, j+1} \leftrightarrow D_{\alpha}^{e j . j+1}=D_{\alpha}^{j, j+1}-\Omega_{\alpha}^{j, j+1} \\
& D_{\alpha}^{j, j+1}=U_{\alpha}^{j+1}-U_{\alpha}^{j}-\frac{e^{j}}{2} \phi_{\alpha}^{j}-\frac{e^{j+1}}{2} \phi_{\alpha}^{j+1} \\
& v^{j . j+1} \leftrightarrow D_{3}^{e j . j+1}=D_{3}^{j, j+1}-\Omega_{3}^{j, j+1} \\
& D_{3}^{j, j+1}=U_{3}^{j+1}-U_{3}^{j}
\end{aligned}
$$

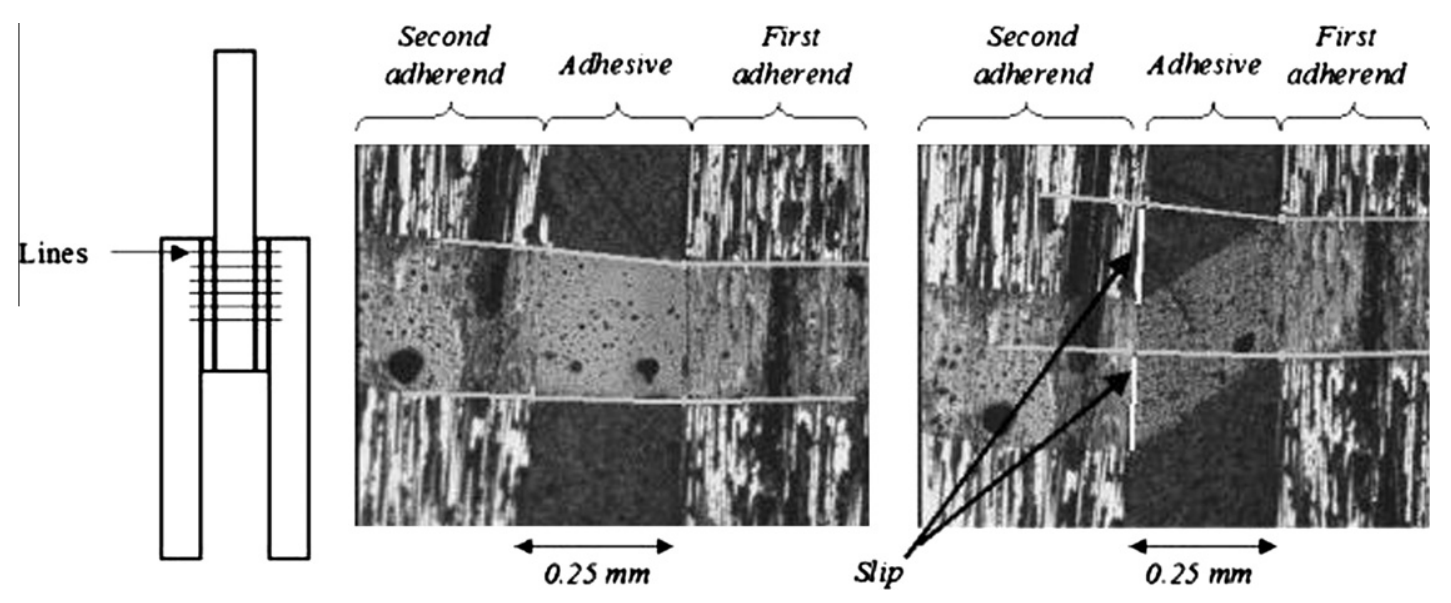

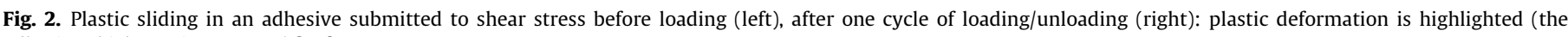
adhesive thickness is $0.25 \mathrm{~mm}$ ) [39]. 
where the $5 n$ following generalized displacements $U_{\alpha}^{i}, \phi_{\alpha}^{i}, U_{3}^{i}$, exhibited by an energetic identification [25], are respectively the generalized in-plane displacement, rotation field and vertical displacement of layer $i$. They result from integrations of the 3D displacement components $u_{1,2,3}(x, y, z)$.

$$
\begin{aligned}
& U_{\alpha}^{i}(x, y)=\int_{h_{i}^{-}}^{h_{i}^{+}} \frac{1}{e^{i}} u_{\alpha}(x, y, z) d z \\
& \Phi_{\alpha}^{i}(x, y)=\int_{h_{i}^{-}}^{h_{i}^{+}} \frac{12}{e^{i^{2}}} u_{\alpha}(x, y, z) d z \\
& U_{3}^{i}(x, y)=\int_{h_{i}^{-}}^{h^{+}} \frac{1}{e^{i}} u_{3}(x, y, z) d z
\end{aligned}
$$

Note that such a set of generalized displacements gives to each layer its own Reissner-Mindlin kinematic. The compatibility conditions are then ensured by the interfaces.

In the previous developments, the interfaces are considered as perfect and interface displacements are only due to the elastic displacements in the neighboring layers. The model made possible to solve free edge elastic problems with singularities [23]. Now, if the role of physical interfaces has to be specifically taken into account, these elastic interface displacements become (see Eq. (2)) the difference between the global interface displacements $D_{\alpha}^{i . j+1}, D_{3}^{i, j+1}$ and the localized slidings due to the own interface behavior $\Omega_{\alpha}^{j, j+1}, \Omega_{3}^{j, j+1}$ which, for instance, can be considered as elastic or plastic.

Then, in this approach, two factors may influence the interfacial rigidity between two layers: the rigidity of adjacent layers and the behavior of interface layer. The role of a thick or/and inelastic adhesive could be then represented by $\Omega_{\alpha}^{j, j+1}, \Omega_{3}^{j, j+1}$. Poor interfacial stiffness or sliding may cause a remarkable decrease of the structure rigidity when a perfect interface (infinite stiffness) provides the expected theoretical stiffness of the structure. It should be noted here and in the rest of the text, that in the finite element formulation, for a convenient numerical reason, purely elastic strain of the interface layer, if it exists (soft elastic connector modeling, for example), will be included in the generalized interlaminar elastic displacements vector composed of the $D_{\alpha}^{e j . j+1}$ and $D_{3}^{e j . j+1}$.

The equilibrium and constitutive equations of the model are then identified with the help of the Hellinger-Reissner variational approach [25]. The five following equations are firstly obtained for each layer $i$ ( $5 n$ equations for all the laminate):

$$
\begin{aligned}
& N_{\alpha \beta, \beta}^{i}+\left(\tau_{\alpha}^{i, i+1}-\tau_{\alpha}^{i-1, i}\right)=0 \\
& Q_{\beta, \beta}^{i}+\left(v^{i, i+1}-v^{i-1, i}\right)=0 \\
& M_{\alpha \beta, \beta}^{i}+\frac{e^{i}}{2}\left(\tau_{\alpha}^{i, i+1}+\tau_{\alpha}^{i-1, i}\right)-Q_{\alpha}^{i}=0
\end{aligned}
$$

Once again, note that this set of $i$ equations is similar to the classical Reissner-Mindlin plate equilibrium equation. The role of interface stresses appears clearly: ensuring equilibrium of all the laminate.

Considering now an orthotropic layer $i$, the 3D compliance $S_{i j k l}^{i}$ can be divided into 3 parts, the in-plane compliance, the transverse shear and normal compliances, described respectively by the following components, $S_{\alpha \beta \gamma \delta}^{i}, S_{\alpha 3 \beta 3}^{i}, S_{33}^{i}$. It leads, by the use of Hellinger-Reissner theorem (details in [25]), to the following elastic constitutive equations:

$$
\begin{aligned}
\varepsilon_{\alpha \beta}^{i}= & \frac{1}{e^{i}} S_{\alpha \beta \gamma \delta}^{i} N_{\gamma \delta}^{i} \\
\chi_{\alpha \beta}^{i} & =\frac{12}{e^{i}} S_{\alpha \beta \gamma \delta}^{i} M_{\gamma \delta}^{i} \\
\gamma_{\alpha}^{i}= & \frac{6}{5 e^{i}} S_{\alpha 3 \gamma 3}^{i} Q_{\beta}^{i}-\frac{1}{10}\left(4 S_{\alpha 3 \gamma 3}^{i}\right)\left(\tau_{\beta}^{i, i+1}+\tau_{\beta}^{i-1, i}\right) \\
D_{\alpha}^{e j . j+1} & =D_{\alpha}^{j, j+1}-\Omega_{\alpha}^{j . j+1}=-\frac{1}{10}\left(4 S_{\alpha 3 \beta 3}^{j}\right) Q_{\beta}^{j}-\frac{1}{10}\left(4 S_{\alpha 3 \beta 3}^{j+1}\right) Q_{\beta}^{j+1}-\frac{e^{j}}{30}\left(4 S_{\alpha 3 \beta 3}^{j}\right) \tau_{\beta}^{j-1, j} \\
& +\frac{2}{15}\left(e^{j}\left(4 S_{\alpha 3 \beta 3}^{j}\right)+e^{j+1}\left(4 S_{\alpha 3 \beta 3}^{j+1}\right)\right) \tau_{\beta}^{j, j+1} \\
& \quad-\frac{e^{j+1}}{30}\left(4 S_{\alpha 3 \beta 3}^{j+1}\right) \tau_{\beta}^{j+1 . j+2} \\
D_{3}^{e j, j+1} & =D_{3}^{j, j+1}-\Omega_{3}^{j, j+1}=\frac{9}{70} e^{j} S_{3333}^{j} \sigma_{3}^{j-1, j}+\frac{13}{35}\left(e^{j} S_{3333}^{j}+e^{j+1} S_{3333}^{j+1}\right) \sigma_{3}^{j, j+1} \\
& +\frac{9}{70} e^{j+1} S_{3333}^{j+1} \sigma_{3}^{j+1, j+2}
\end{aligned}
$$

\subsection{Interface with its own plastic model}

In the following part, a plastic behavior of the interface is assumed. It will precise the form of $\Omega_{\alpha}^{j, j+1}$ and $\Omega_{3}^{j, j+1}$ in the fourth and fifth equations of Eq. (5). The interface layer has a thickness $e^{j j^{j+1}}$, a Young modulus $E^{j+1}$ and a Poisson ratio $v^{j, j+1}$. Based on the classical hypothesis that the adhesive thickness is weak as compared to layers thickness, only out-of-plane shear stresses and normal stress are taken into account. The interlaminar stress and strains are assumed to be constant through the adhesive thickness. To complete the multilayer modeling behavior (Eq. 5), the interlaminar slips $\Omega_{\alpha}^{j, j+1}$ and $\Omega_{3}^{j, j+1}$ have to be stated. They reflect the physical nature of the interface.

With previous assumptions and definitions, the integration of the $3 \mathrm{D}$ strains leads to the following equations:

$$
\begin{aligned}
& 2 e^{j, j+1} \varepsilon_{31}^{j . j+1}(x, y)=\int_{e^{j, j+1}}\left(u_{1,3}+u_{3,1}\right)(x, y, z) d z=\Omega_{1}^{j, j+1}(x, y)+g_{1}^{j, j+1}(x, y) \\
& 2 e^{j, j+1} \varepsilon_{32}^{j, j+1}(x, y)=\int_{e^{j, j+1}}\left(u_{2,3}+u_{3,2}\right)(x, y, z) d z=\Omega_{2}^{j, j+1}(x, y)+g_{2}^{j, j+1}(x, y) \\
& e^{j, j+1} \varepsilon_{33}^{j, j+1}(x, y)=\int_{e^{j, j+1}} u_{3,3}(x, y, z) d z=\Omega_{3}^{j, j+1}(x, y)
\end{aligned}
$$

where

$g_{1}^{j . j+1}(x, y)=\int_{e^{j, j+1}} u_{3,1}(x, y, z) d z$

and

$g_{2}^{j, j+1}(x, y)=\int_{e^{j, j+1}} u_{3,2}(x, y, z) d z$.

As proposed in [27], the integration of the 3D displacement component $u_{3}(x, y, z)$ at the interface can be interpolated through the adjacent layer generalized displacements $U_{3}^{j}(x, y)$, as defined in Eq. 3. So, the following equations are deduced:

$\left.g_{1}^{j, j+1}(x, y)=e^{j, j+1} \frac{U_{3,1}^{j}(x, y) e^{j+1}+U_{3,1}^{j+1}(x, y) e^{j}}{e^{j}+e^{j+1}}\right)$
$\left.g_{2}^{j, j+1}(x, y)=e^{j, j+1} \frac{U_{3,2}^{j}(x, y) e^{j+1}+U_{3,2}^{j+1}(x, y) e^{j}}{e^{j}+e^{j+1}}\right)$

The interface layer is assumed to be isotropic. From 6 the constitutive equations become:

$$
\begin{aligned}
& \Omega_{1}^{j, j+1}=\frac{2 e^{j, j+1}\left(1+\nu^{j, j+1}\right)}{E^{j, j+1}} \tau_{1}^{j, j+1}-g_{1}^{j, j+1}(x, y)+\Omega_{1}^{j, j+1^{p}}(x, y) \\
& \Omega_{2}^{j, j+1}=\frac{2 e^{j, j+1}\left(1+\nu^{j, j+1}\right)}{E^{j, j+1}} \tau_{2}^{j, j+1}-g_{2}^{j, j+1}(x, y)+\Omega_{2}^{j, j+1^{p}}(x, y) \\
& \Omega_{3}^{j, j+1}=\frac{e^{j, j+1}}{E^{j, j+1}} \sigma_{3}^{j, j+1}+\Omega_{3}^{j, j+1^{p}}(x, y)
\end{aligned}
$$

where $\Omega_{1}^{j \cdot j+1^{p}}(x, y), \Omega_{2}^{j . j+1^{p}}(x, y), \Omega_{3}^{j . j+1^{p}}(x, y)$ are the plastic displacement discontinuities due to the interface, if they exist. If they do not, this expression can also describe a localized purely elastic behavior of the interface, as in the problem of connections between media, with soft interfaces. In [2] such an approach was successfully used to predict the typical behavior of a concrete-wood composite beam with discrete connectors.

If there are non-elastic slidings at the interface, $\Omega_{1}^{j . j+1^{p}}(x, y)$, $\Omega_{2}^{j, j+1^{p}}(x, y), \Omega_{3}^{j, j+1^{p}}(x, y)$ can be classically described by:

- A Von-Mises criterion

$$
f(\boldsymbol{\sigma})=\sigma_{e q}^{j, j+1}-\sigma_{c r}^{j, j+1} \leqslant 0
$$

where

$$
\sigma_{e q}^{j . j+1}=\sqrt{\sigma_{3}^{j . j+1^{2}}+3\left(\tau_{1}^{j . j+1^{2}}+\tau_{2}^{j . j+1^{2}}\right)}
$$

and $\sigma_{c r}^{j . j+1}$ is a constant critical stress. Any other criteria can be considered, as a Drucker-Prager one for a confined situation [26] for instance. 
- A 3D plastic flow rule

$$
\begin{aligned}
& \dot{\Omega}_{1}^{j, j+1^{p}}=3 e^{j, j+1} \dot{p}^{j, j+1} \frac{\tau_{1}^{j, j+1}}{\sigma_{c r}^{j, j+1}} \\
& \dot{\Omega}_{2}^{j, j+1^{p}}=3 e^{j, j+1} \dot{p}^{j, j+1} \frac{\tau_{2}^{j, j+1}}{\sigma_{c r}^{j, j+1}} \\
& \dot{\Omega}_{3}^{j, j+1^{p}}=e^{j, j+1} \dot{p}^{j, j+1} \frac{\sigma_{3}^{j, j+1}}{\sigma_{c r}^{j, j+1}}
\end{aligned}
$$

if $f(\boldsymbol{\sigma})=0, \dot{f}(\boldsymbol{\sigma})=0$ and $\dot{p}^{j, j+1}>0$, where $p^{j, j+1}$ is the cumulative plastic strain at the interface $j, j+1$.

Diaz et al. [27] cuidado propose a resolution of the equations by applying the LATIN (LArge Time INcrement) method introduced by Ladeveze [40] for a double lap bonding problem.

In the following part, the paper introduces the development of the finite element model MPFEAP for such an interface behavior.

\section{Multiparticle finite element model}

Based on the previous development, a $\mathbb{C}^{0}$ finite element model, involving an eight-node isoparametric quadrilateral element with $5 n$ degrees of freedom at each nodal point and four second-order Gaussian points, is formulated. A program called MPFEAP (Multiparticle Finite Element Analysis Program) has been developed for the implementation of the proposed element in [24] which permits to solve static laminated elastic plate problems. A recent development uses the mixed algorithm subspace algorithm proposed by Dhatt and Touzot [41] to calculate $p$ first vibration modes. The present development includes the elastoplastic interface introduced in the previous section.

\subsection{Geometry and displacement interpolations}

The square element defined in the $\xi, \eta$ space is shown in Fig. 3.

It is based on eight nodal points and the shape functions are described in [41].

The $5 n$ ( $n$ stands for the number of layers) displacement unknown vector of $i$ th node is defined as

$\boldsymbol{\delta}_{i}^{T}=\left\langle U_{i 1}^{1} U_{i 2}^{1} U_{i 3}^{1} \phi_{i 1}^{1} \phi_{i 2}^{1} \cdots U_{i 1}^{n} U_{i 2}^{n} U_{i 3}^{n} \phi_{i 1}^{n} \phi_{i 2}^{n}\right\rangle$

where $U_{i 1}^{k}$ and $\phi_{i 2}^{k}$ are the generalized displacements defined in Eq. (3).

\subsection{Strain interpolation and associated stress}

The $11 n-3$-dimension strain vector $\epsilon$ is expressed with the generalized strains defined in Eq. 2 and by separating the components concerning the $6 n$ membrane strain vector $\epsilon^{c}$, the $(n-1)$ normal strain and the $(4 n-2)$ shear strain vector $\epsilon^{Q}$ as follows:

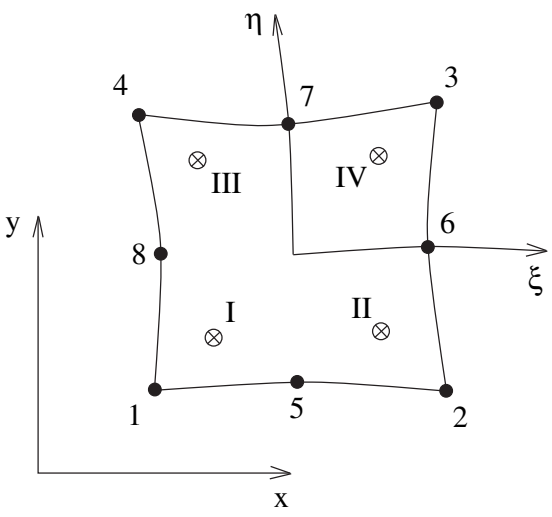

Fig. 3. The eight-node element and its four second-order Gaussian station.

$$
\begin{aligned}
& \boldsymbol{\epsilon}^{T}\left\langle\boldsymbol{\epsilon}^{c}, \boldsymbol{\epsilon}^{Q}\right\rangle \\
& \boldsymbol{\epsilon}^{c}=\left\langle\varepsilon_{11}^{1} \varepsilon_{22}^{1} 2 \varepsilon_{12}^{1} \chi_{11}^{1} \chi_{22}^{1} 2 \chi_{12}^{1} \ldots \varepsilon_{11}^{n} \varepsilon_{22}^{n} 2 \varepsilon_{12}^{n} \chi_{11}^{n} \chi_{22}^{n} 2 \chi_{12}^{n}\right\rangle^{T} \\
& \boldsymbol{\epsilon}^{Q}=\left\langle\gamma_{1}^{1} \gamma_{2}^{1} D_{1}^{1,2} D_{2}^{1,2} D_{3}^{1,2} \gamma_{1}^{2} \gamma_{2}^{2} \ldots \gamma_{1}^{n-1} \gamma_{2}^{n-1} D_{1}^{n-1, n} D_{2}^{n-1, n} D_{3}^{n-1, n} \gamma_{1}^{n} \gamma_{2}^{n}\right\rangle^{T}
\end{aligned}
$$

So, the 3-dimension generalized elementary interlaminar strain vector can be organized as follows:

$\epsilon^{j, j+1}=\left\langle D_{1}^{j, j+1} D_{2}^{j, j+1} D_{3}^{j . j+1}\right\rangle$

The 3 components of the slip vector due to the plastic deformation of the interface $j, j+1$ as defined in Eq. 11 are compiled in $\boldsymbol{\Omega}^{p \mathrm{j} j+1}$ :

$\mathbf{\Omega}^{p j, j+1}=\left\langle\Omega_{1}^{j, j+1}{ }^{p} \Omega_{2}^{j, j+1}{ }^{p} \Omega_{3}^{j, j+1} p\right.$

The associated stress vector is expressed similarly, including the generalized forces as previously defined in Eq. 1:

$$
\begin{aligned}
& \boldsymbol{\sigma}^{T}=\left\langle\boldsymbol{\sigma}^{c}, \boldsymbol{\sigma}^{Q}\right\rangle \\
& \boldsymbol{\sigma}^{c}=\left\langle N_{11}^{1} N_{22}^{1} N_{12}^{1} M_{11}^{1} M_{22}^{1} M_{12}^{1} \cdots N_{11}^{n} N_{22}^{n} N_{12}^{n} M_{11}^{n} M_{22}^{n} M_{12}^{n}\right\rangle^{T} \\
& \boldsymbol{\sigma}^{Q}=\left\langle Q_{1}^{1} Q_{2}^{1} \tau_{1}^{1,2} \tau_{2}^{1,2} \sigma_{3}^{1,2} Q_{1}^{2} Q_{2}^{2} \cdots Q_{1}^{n-1} Q_{2}^{n-1} \tau_{1}^{n-1, n} \tau_{2}^{n-1, n} \sigma_{3}^{n-1, n} Q_{1}^{n} Q_{2}^{n}\right\rangle^{T}
\end{aligned}
$$

\subsection{Stress-strain relation}

The M4-5n generalized strains and stresses relationship can be expressed in the matrix form as

$\boldsymbol{\epsilon}=\boldsymbol{S} \boldsymbol{\sigma}+\boldsymbol{\epsilon}^{0}$

where the compliance matrix $\boldsymbol{S}$ of dimension $(11 n-3) \times(11 n-3)$ is composed of the in plane compliance matrix $\boldsymbol{S}_{6 n \times 6 n}^{p}$ (in plane components $S_{\alpha \beta \gamma \delta}^{i}$ of the layer $i$ ), the normal and shear compliance $\left(S_{\alpha 3 \beta 3}^{i}, S_{3333}^{i}\right)$ compiled in the matrix $\boldsymbol{S}_{(5 n-3) \times(5 n-3)}^{Q}$

$\boldsymbol{S}=\left(\begin{array}{cc}\boldsymbol{S}^{p} & \mathbf{0} \\ \mathbf{0} & \boldsymbol{S}^{Q}\end{array}\right)$

The initial strain vector $\epsilon^{0}$ is due to the load $T^{+}$and $T^{-}$given on the top and the bottom surfaces. The elastic constitutive equation in stiffness terms can be computed as

$\boldsymbol{\sigma}=\boldsymbol{S}^{-1}\left(\boldsymbol{\epsilon}-\boldsymbol{\epsilon}^{0}\right)=\boldsymbol{D}\left(\boldsymbol{\epsilon}-\boldsymbol{\epsilon}^{0}\right)$

- In the elastic case (it means with infinitely rigid interfaces), matrix $\boldsymbol{D}$ of dimension $(11 n-3) \times(11 n-3)$ is simply defined by

$$
\left.\boldsymbol{D}=\boldsymbol{D}^{e l}=\left(\begin{array}{cc}
\boldsymbol{D}^{p} & \mathbf{0} \\
\mathbf{0} & \boldsymbol{D}^{Q}
\end{array}\right)=\begin{array}{cc}
\boldsymbol{S}^{p^{-1}} & \mathbf{0} \\
\mathbf{0} & \boldsymbol{S}^{Q^{-1}}
\end{array}\right)
$$

- In the elastic case with elastic sliding at interfaces, for a convenient numerical reason, purely elastic behaviors of the interfaces are included in the out-of-plane part $\boldsymbol{D}^{Q}$ of the above elastic matrix $\boldsymbol{D}^{e l}$.

- In the elastic case with plastic sliding at interfaces, the stiffness matrix is reduced and calculated by:

$$
\boldsymbol{D}=\boldsymbol{D}^{e l}-\boldsymbol{D}^{c o r}
$$

where, the correction matrix $\boldsymbol{D}^{\text {cor }}$ has non-zero terms corresponding to the plastic interfaces which are deduced by retour radial algorithm (or projected algorithm). So, in the following section, the $\boldsymbol{D}^{\text {cor }}$ will be the correction matrix of the elastoplastic interface.

\subsection{Adaptation of a radial return algorithm}

The integration algorithm called Radial Return Algorithm is widely used for nonlinear analysis of structures. The load is 
discretized. At each time step, the system state is calculated based on the state of previous time step. To do this, local and global iterations are calculated until the convergence condition is satisfied. Because of some neglected stress terms in the matrix of the adhesive, the algorithm must be adapted to this model: all the stress and strains terms neglected in the theory are not taken into account in the algorithm formulation.

1. At $n+1$ th time step, the elastic predictor: $\boldsymbol{\sigma}_{n+1}^{\text {elas }}=\left[\begin{array}{lll}\tau_{1} & \tau_{2} & \sigma_{3}\end{array}\right]^{T}$ is calculated from the closest state calculated: strain $\epsilon_{n+1}$, plastic deformation of the interface of $n$th time step $\boldsymbol{\Omega}_{n}^{p}$ and plastic deformation increased at $n+1$ th time step calculated at $k$ th iteration $\Delta \boldsymbol{\Omega}_{n}^{p, k}$

2. Calculate $\sigma_{n+1}^{\text {elas,eq }}=\sqrt{\sigma_{3}^{2}+3 \tau_{1}^{2}+3 \tau_{2}^{2}}$

3. Estimate

$$
f_{n+1}^{\text {elas }}=f\left(\boldsymbol{\sigma}_{n+1}^{\text {elas }}, p_{n}\right)=\sigma_{n+1}^{\text {elas,eq }}-R\left(p_{n}\right)
$$

where $R\left(p_{n}\right)=\sigma_{c r}$ is a critical constant strength in the case of perfect plasticity

(a) If $f_{n+1}^{\text {elas }} \leqslant 0$, update the cumulative plastic strain, plastic tensor and plastic deformation increased

$$
p_{n+1}=p_{n}, \quad \boldsymbol{D}^{\text {cor }}=0, \quad \Delta \boldsymbol{\Omega}_{n}^{p, k+1}=\Delta \boldsymbol{\Omega}_{n}^{p, k}
$$

(b) If $f_{n+1}^{\text {elas }}>0$ :

(i) Solve $\Delta p_{n}$ satisfying:

$\sigma_{n+1}^{\text {elas,eq }}-3 \mu \Delta p_{n}-R\left(p_{n}+\Delta p_{n}\right)=0$

where $\mu$ is the Lame coefficient.

(ii) Refresh cumulative plastic strain:

$p_{n+1}=p_{n}+\Delta p_{n}$

(iii) Evaluate $\beta$ and $\gamma$ constants:

$\beta=\frac{3 \mu \Delta p_{n}}{\sigma_{n+1}^{\text {elas }, q}} \quad \gamma=\frac{3 \mu}{3 \mu+R_{n+1}^{\prime}}$

(iv) Update plastic strain increment:

$$
\begin{gathered}
\delta \boldsymbol{\Omega}_{n+1}^{p}=\frac{3 \Delta p_{n}}{2 \sigma_{n+1}^{\text {elas,eq }}}\left[\begin{array}{c}
\tau_{1} \\
\tau_{2} \\
\frac{2}{3} \sigma_{3}
\end{array}\right] \\
\Delta \boldsymbol{\Omega}_{n+1}^{p, k+1}=\Delta \boldsymbol{\Omega}_{n+1}^{p, k}+\delta \boldsymbol{\Omega}_{n+1}^{p}
\end{gathered}
$$

(v) Correct plastic tensor $\boldsymbol{D}^{\text {cor }}$ :

$$
\boldsymbol{D}^{\text {cor }}=\frac{3 \mu(\gamma-\beta)}{e\left(\sigma_{n+1}^{\text {elas }, e q}\right)^{2}}\left[\begin{array}{ccc}
\frac{4}{9} \sigma_{3}^{2} & \frac{2}{3} \sigma_{3} \tau_{2} & \frac{2}{3} \sigma_{3} \tau_{1} \\
\frac{2}{3} \sigma_{3} \tau_{2} & \tau_{2}^{2} & \tau_{1} \tau_{2} \\
\frac{2}{3} \sigma_{3} \tau_{1} & \tau_{1} \tau_{2} & \tau_{1}^{2}
\end{array}\right]+\frac{2 \mu \beta}{e}\left[\begin{array}{ccc}
\frac{2}{3} & 0 & 0 \\
0 & \frac{1}{2} & 0 \\
0 & 0 & \frac{1}{2}
\end{array}\right]
$$

\section{Example and numerical results}

The relevance of the $2 \mathrm{D}$ finite element is studied through a comparison with a 3D finite element method (ABAQUS). The case study is a double lap joint. In this example, interlaminar stresses and slips are considered.

The double lap joint is $124 \mathrm{~mm}$ long and $16.2 \mathrm{~mm}$ wide. A uniform tension is applied (Fig. 4) and the joint is calculated by MPFEAP and the 3D model of ABAQUS. Note that interlaminar shear stress $\tau_{1}$ and interlaminar normal stress $\sigma_{3}$ correspond, respectively, to $\sigma_{x z}$ et $\sigma_{z z}$ of 3D stress tensor of joint layer. Due to the symmetry, only one quarter of the joint is studied (shaded region). The material properties are:

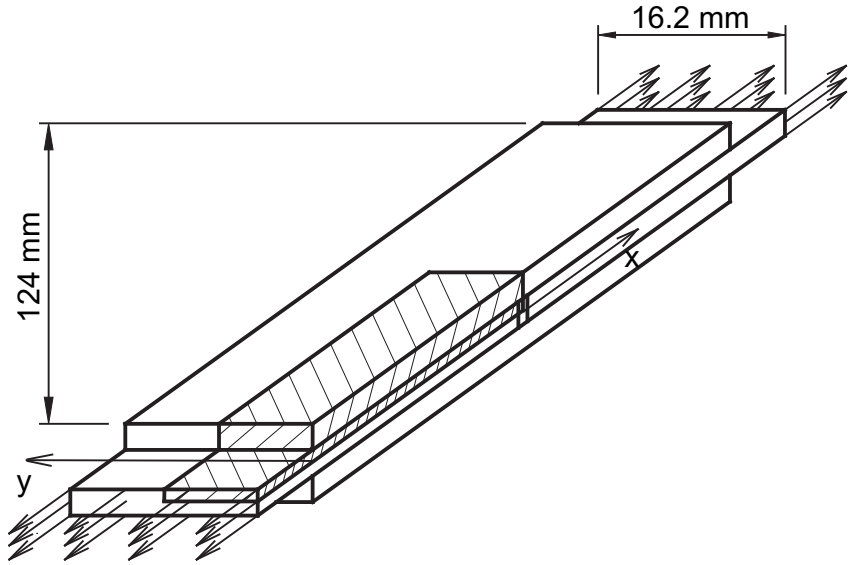

Fig. 4. Double lap joint studied.

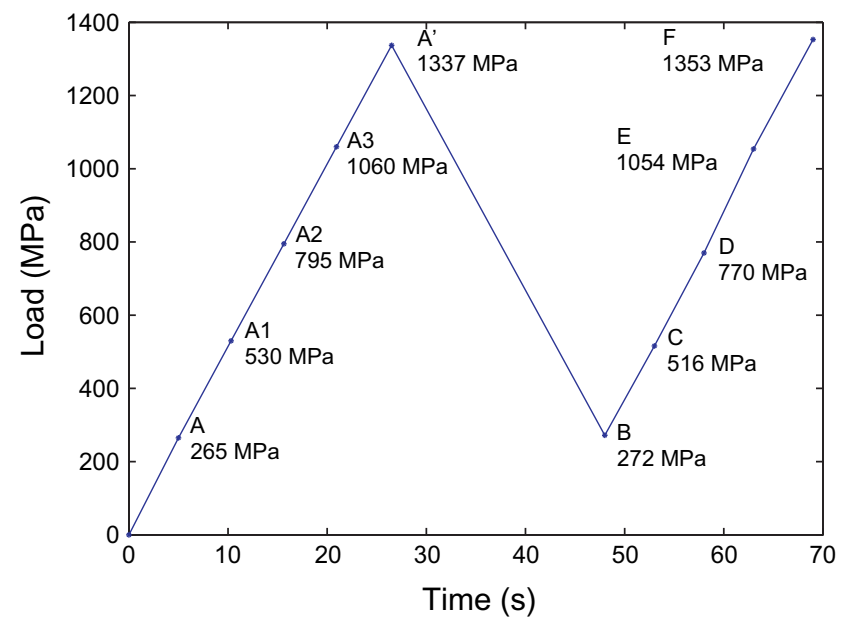

Fig. 5. Load-unload-load scheme.

Adherent composite with carbon fiber: $E_{1}=E_{2}=E_{3}=162,000$ MPa, $v_{12}=v_{13}=v_{23}=0.3, e=1.2 \mathrm{~mm}$ Adhesive layer: $E=2500 \mathrm{MPa}$, $v=0.3, e=0.25 \mathrm{~mm}$, critical stress $\sigma_{c r}=37 \mathrm{MPa}$

The tension scheme is a load-unload-load scheme (Fig. 5). The interfacial stresses and slips at load points A (265 MPa), $A^{\prime}$ (1337 MPa), B (272 MPa), C (516 MPa), D (770 MPa), E (1054 MPa) and $\mathrm{F}(1353 \mathrm{MPa})$ are compared. In order to model the shaded region of the double lap joint with MPFEAP (Fig. 4), different plane meshes are used: 8 by 20 and 10 by 40 elements, and each 2D element integrates adherents and adhesives. Each mesh is refined near the edges (Figs. 6 and 7). These meshes predict well the plasticity beginning at the edges. The mesh $10 \times 40$ satisfies the interlaminar stress and slip convergence.

To model the joint with ABAQUS, a mesh of 123 by 20 elements describes the plane geometry. Four elements in thickness of each layer and 8 elements in thickness of adhesive layer are used and necessary to ensure the convergence of calculations. The interlaminar stresses of model $\tau_{1}, \tau_{2}, \sigma_{3}$ are compared to $\sigma_{33}, \sigma_{x z}, \sigma_{y z}$ of the 3D model provided by ABAQUS in the middle of the adhesive layer. The interlaminar slips $\Omega_{1}, \Omega_{2}, \Omega_{3}$ provided by ABAQUS are the difference between the ABAQUS displacements of the superior adhesive surface and the lower adhesive surface.

Compared to the 3D method, the proposed layerwise 2D model advantages are: 


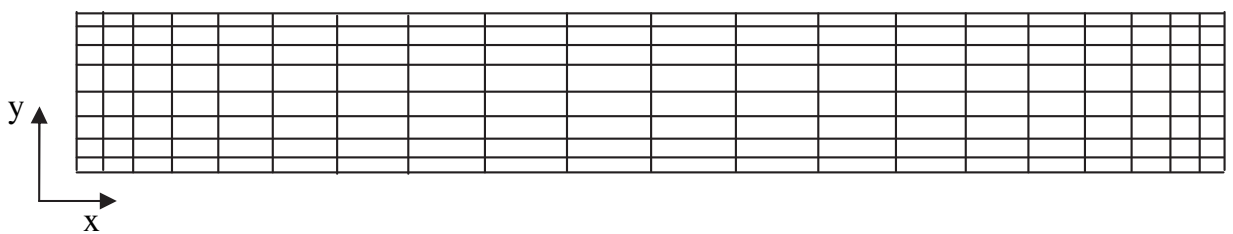

Fig. 6. $8 \times 20$ mesh

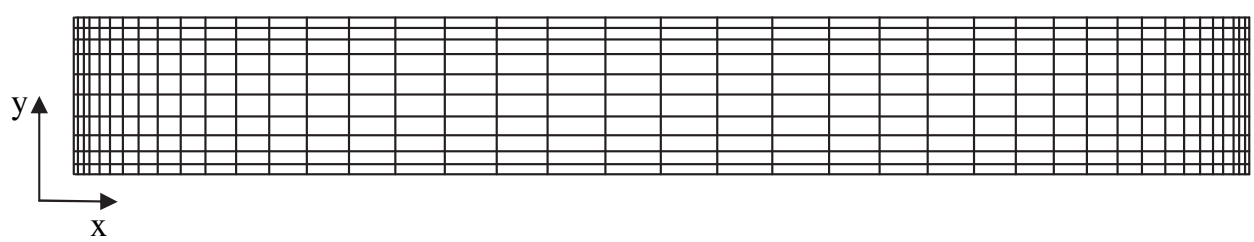

Fig. 7. $10 \times 40$ mesh.

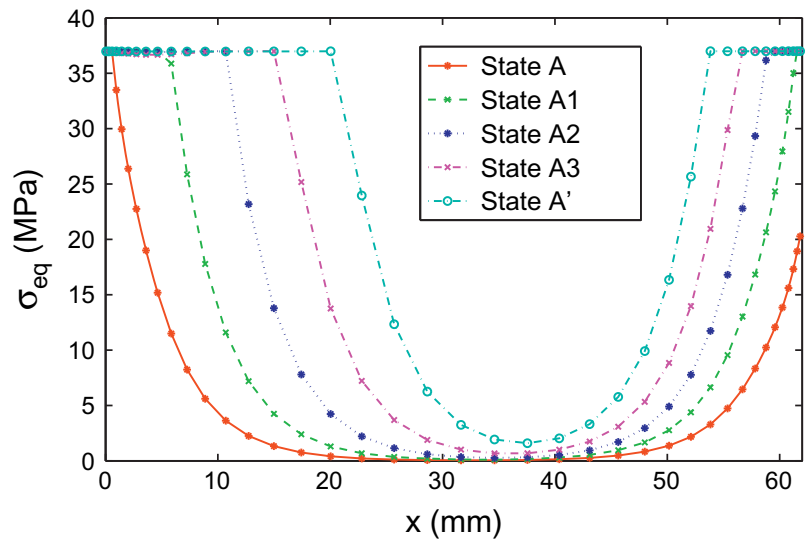

Fig. 8. Plasticity propagation from A to $A^{\prime}$.
- The necessary number of degree of freedom for the M4-5n model is very smaller than that for the 3D model. (2685 degrees of freedom for the $8 \times 20$ mesh and 5155 degrees of freedom for the $10 \times 40$ mesh, 265,608 degrees of freedom for the ABAQUS mesh).

- Due to the compact vision (integration with respect to $z$ ) of the multilayer, the M4-5n model provides relevant finite results even on the edges (details in [42]). By contrast, the 3D method results are generally singular on the edges. In this case, the plasticity smoothes these singularities in the 3D calculation and provides finite and converged values.

- The M4-5n model does not need a through the thickness discretization even to obtain interface values. By contrast, the quality of the through the thickness discretization is very determinant and computationally expensive in the 3D method.

To discuss the numeric results obtained by MPFEAP, the equivalent stress, generalized stresses, and slidings of the interface are

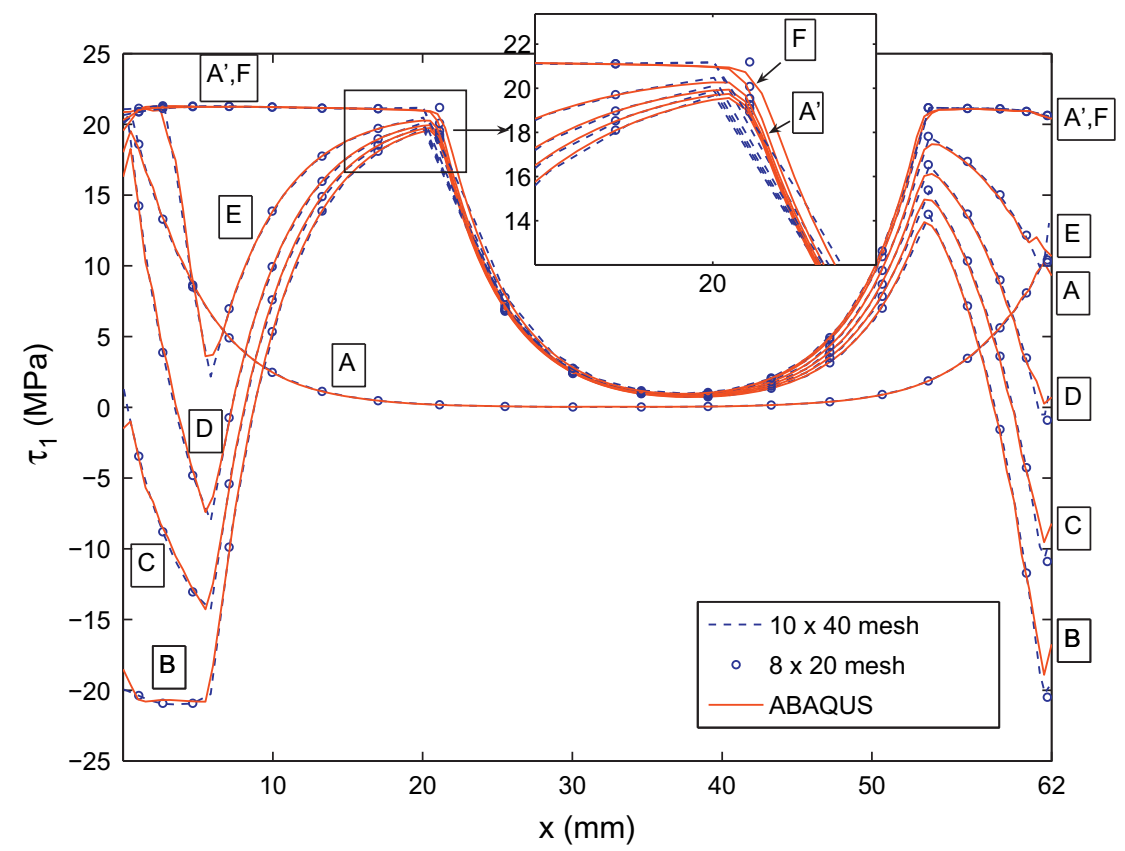

Fig. 9. Shear stress $\tau_{1}$ during the load-unload-load cycle. 


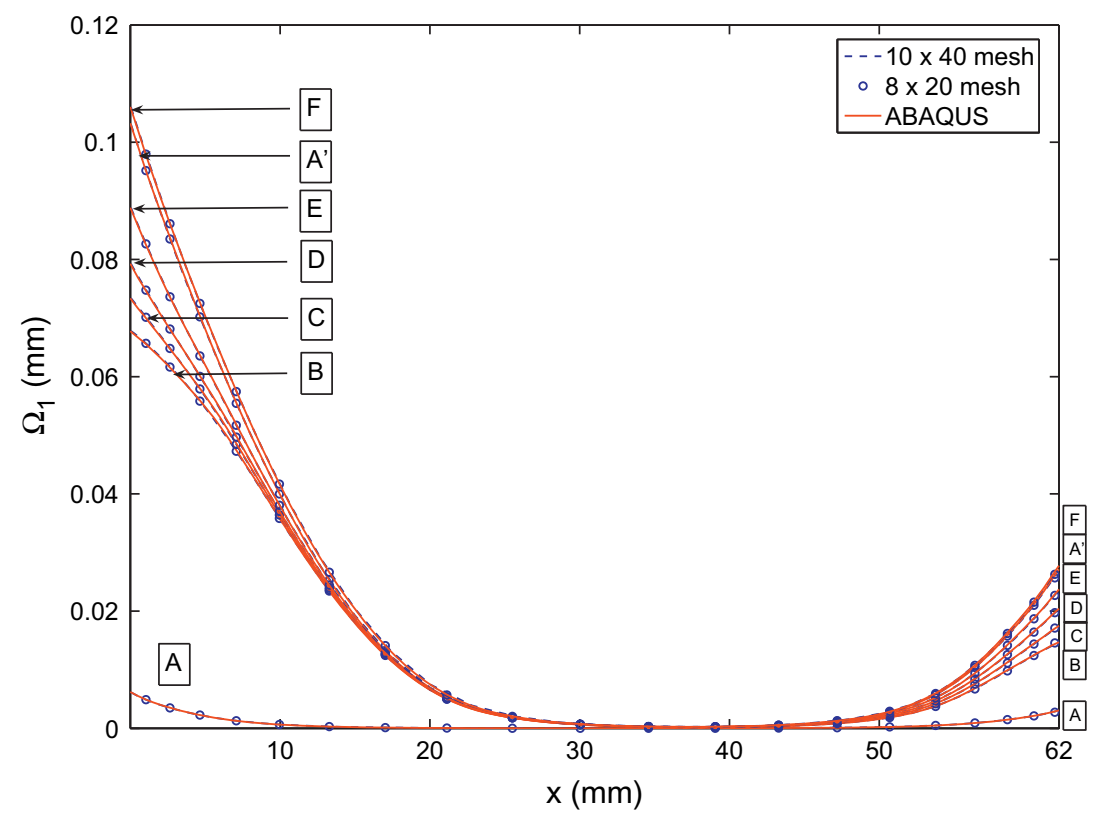

Fig. 10. Slip $\Omega_{1}$ during the load-unload-load cycle.

considered. Equivalent stress $\sigma_{e q}=\sqrt{\sigma_{3}^{2}+3 \tau_{1}^{2}+3 \tau_{2}^{2}}$ is calculated at the reference loading points $\mathrm{A}, \mathrm{A} 1, \mathrm{~A} 2, \mathrm{~A} 3, \mathrm{~A}^{\prime}$ (Fig. 8). These points correspond respectively to $265 \mathrm{MPa}, 530 \mathrm{MPa}, 795 \mathrm{MPa}$, $1060 \mathrm{MPa}$ and $1337 \mathrm{MPa}$. At load point $\mathrm{A}$, the first elements near the edge begin to be plasticized. From $A$ to $A^{\prime}$, plastic zone propagates (Fig. 8). From $A^{\prime}$ to $F$, stresses and interfacial generalized displacements will be compared to the results obtained by the $3 \mathrm{D}$ model. The shear stress $\tau_{1}$ obtained by the present model during all the cycle is presented in Fig. 9. The results of the $8 \times 20$ mesh and of the $10 \times 40$ mesh correspond to those of ABAQUS. The interlaminar transverse slip $\Omega_{1}$ (Fig. 10) is also very well approximated.
In Fig. 11, the interlaminar normal stress $\sigma_{3}$ obtained by the M4-5n model approximation is shown. The results are very close despite a chaotic nature of the stress field. The interlaminar normal slip $\Omega_{3}$ comparison presented in Fig. 12 is very satisfactory, too. In brief, from Figs. 9-12, it is clear that MPFEAP and the 3D finite element produce almost the same interlaminar slip and stresses during all the load cycle. It means that the interlaminar generalized stresses in M4-5n are the average 3D value obtained by the 3D finite element model, and the interlaminar generalized slip in M4-5n is the 3D slip obtained by 3D model. The mesh in the 3D finite element model will be more refined in the case the adhesive thickness
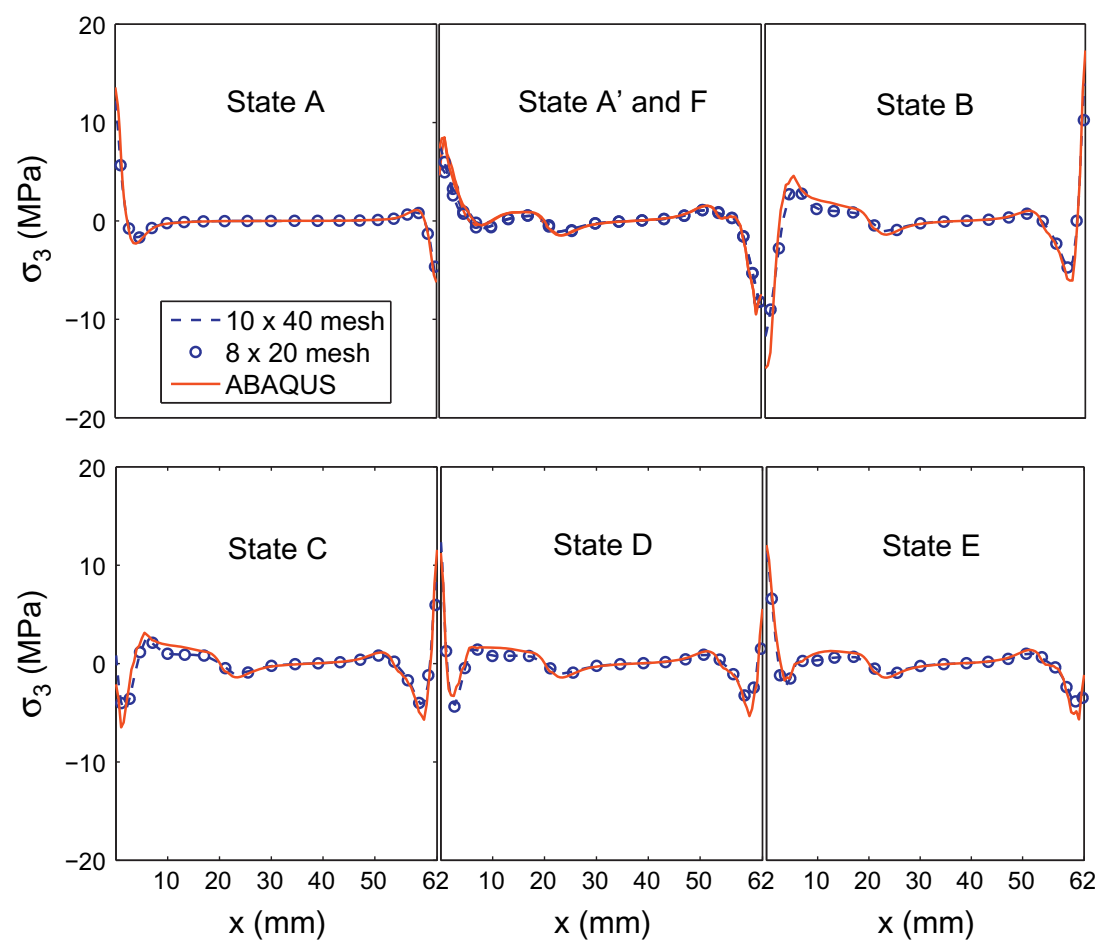

Fig. 11. Normal stress $\sigma_{3}$ during the load-unload-load cycle. 

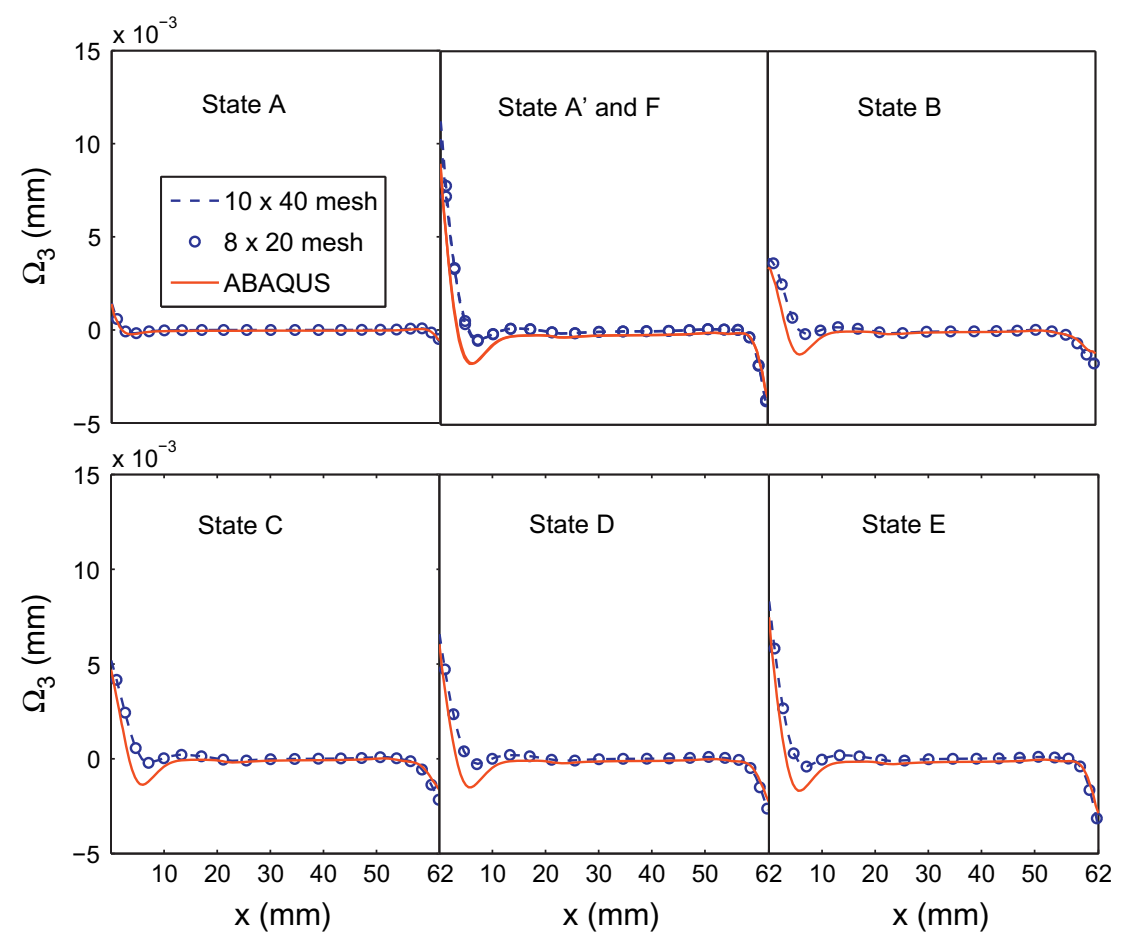

Fig. 12. Normal slip $\Omega_{3}$ during the load-unload-load cycle.

reduces. By contrast, the mesh in MPFEAP is not under the influence of the adhesive thickness. As for this case $\left(e_{\text {adherent }}=1.2 \mathrm{~mm}\right.$, $e_{\text {adhesive }}=0.25 \mathrm{~mm}$ ), the calculation time of ABAQUS is much longer than the MPFEAP one, $7 \mathrm{~h}$ as compared to $25 \mathrm{~min}$.

\section{Conclusion}

In this paper, a $2 \mathrm{D}$ plane representation of a multilayer with imperfect interface is proposed. The mechanical approach belongs to the layerwise model family. The 3D stresses are approximated through the thickness by polynomial expressions consistent with 3D equilibrium conditions. By an energetic balance principle, a plate kinematic is associated to each layer providing interface conditions, which are necessary to respect the multilayer global equilibrium. Such an approach makes possible to describe directly the interface local phenomena and to determine the level of stresses, and the elastic or plastic sliding which can be found in connected or bonded systems. A comparison with a $3 \mathrm{D}$ calculation is proposed for a load-unload double lap elastoplastic joint. The results are very satisfactory, and the following benefits of the plate approach are highlighted:

- Since the thickness is not meshed (plate approach), the mesh is in MPFEAP much simpler than that in a 3D classical finite element model approach, and, as a consequence, the number of degrees of freedom is significantly reduced. In this application, for only one plastic interface, a ratio of $1 / 100$ is shown between the number of nodes of MPFEAP and ABAQUS. Obviously, in the case of a multilayer with several interfaces, this ratio decreases even more with the number of interfaces.

- The computation time is much lower.

- Due to the specific 2D compacted vision of the laminate, results are not singular on the edges, and therefore, elastic or plastic delamination stress criteria can be proposed. This has been done in [1] for studying delamination in carbon-epoxy composites. The confrontation between an analytical approach of the free edge problem and an experimental campaign have validated such a stress criteria, which can be related to toughness or energetic considerations.

The implementation of this element in Abaqus is underway.

\section{References}

[1] Diaz AD, Caron JF. Interface plasticity and delamination onset prediction. Mech Mater 2006;38:648-63.

[2] Roy RL, Pham H, Foret G. New wood composite bridges. Eur J Environ Civil Eng 2009; 13:1125-40.

[3] Miot S, Hochard C, Lahellec N. A non-local criterion for modelling unbalanced woven ply laminates with stress concentrations. Compos Struct 2010;92:1574-80.

[4] Leguillon D, Marion G, Harry R, LTcuyer F. The onset of delamination at stressfree edges in angle-ply laminates - analysis of two criteria. Compos Sci Technol 2001;61(3):377-82.

[5] Brewer J, Lagace P. Quadratic stress criterion for initiation of delamination. J Compos Mater 1988;22:1141-55.

[6] Carrera E. Theories and finite elements for multilayered, anisotropic, composite plates and shells. Arch Comput Methods Eng 2002;9(2):87-140.

[7] Reddy J. Mechanics of laminated composite plates, theory and analysis. CRC Press; 1997.

[8] Reissner E, Stavsky Y. Bending and stretching of certain types of heterogenous aelotropic elastic plates. ASME J Appl Mech 1961;28:402-9.

[9] Reissner E. The effect of transverse shear deformation on the bending of elastic plates. ASME J Appl Mech 1945;12(2):69-77.

[10] Mindlin R. Influence of rotatory inertia and shear on flexural motions of isotropic elastic plates. ASME J Appl Mech 1951;18:31-8.

[11] Lebée A, Sab K. A cosserat multiparticle model for periodically layered materials. Mech Res Commun 2010;37(3):293-7.

[12] D'Ottavio M, Ballhause D, Wallmersperger T, Kroplin B. Considerations on higher-order finite elements for multilayered plates based on a unified formulation. Comput Struct 2006;84:1222-35.

[13] Ferreira AJM. formulation of the multiquadric radial basis function method for the analysis of laminated composite plates. Compos Struct 2003;59(3):385-92.

[14] Carrera E. Historical review of zig-zag theories for multilayered plates and shells. Appl Mech Rev 2003;56:287-308.

[15] Toledano A, Murakami H. A high-order laminated plate theory with improved in-plane responses. Int J Solids Struct 1987;23:111-31.

[16] Carrera E. CO Reissner-Mindlin multilayered plate elements including zig-zag and interlaminar stress continuity. Int $\mathrm{J}$ Numer Methods Eng 1996;39:1797-820. 
[17] Polit O, Touratier M. A multilayered/sandwich triangular finite element applied to linear and non-linear analyses. Compos Struct 2002;58(1):121-8.

[18] Srinivas S. A refined analysis of composite laminates. J Sound Vib 1973;30:495-507.

[19] Carrera E. A class of two-dimensional theories for anisotropic multilayered plates analysis, Accademia delle Scienze di Torino. Memorie Scienze Fisiche 1995-1996;19-20:1-39.

[20] Pagano N. Stresses fields in composite laminates. Int J Solids Struct 1978;14:385-400.

[21] Reissner E. On a variational theorem in elasticity. J Math Phys 1950;29:90-5.

[22] Naciri T, Chabot A, Ehrlacher A. Interlaminar stress analysis with a new multiparticle modelization of multilayered materials (m4). Compos Sci Technol 1998;58:337-79.

[23] Caron JF, Diaz AD, Chabot A, Ehrlacher A. Multi-particle modelling for the prediction of delamination in multi-layered materials. Compos Sci Technol 2006;66:755-65.

[24] Nguyen V, Caron JF. A new finite element for free edge effect analysis in laminated composites. Comput Struct 2006;84:1538-46.

[25] Carreira R, Caron JF, Diaz AD. Model of multilayered materials for interface stresses estimation and validation by finite element calculations. Mec Mater 2002;34:217-30.

[26] Chataigner S, Caron JF. Optimisation of the shear stress transfer in structura bonded assemblies using a curved bonded joint geometry. Construct Build Mater 2011;25(2):442-51

[27] de los Rios G, Balderas R, Duong V, Caron JF, Ehrlacher A, Foret G, et al Laminated materials with plastic interfaces: modeling and calculation. Model Simul Mater Sci Eng 2009;17(2).

[28] Papanicolaou G, Bakos D, Kosmidou T. Effect of the interface stiffness and skincore adhesion efficiency on the interfacial stress distribution of sandwich structures. Composites: Part A 2007;38:1099-106.
[29] Allix O, Ladevèze P. Interlaminar interface modelling for the prediction of laminate delamination. Compos Struct 1992;22:235-42.

[30] Needleman A. An analysis of tensile decohesion along an interface. J Mech Phys Solids 1989;38:289-324.

[31] Tvergaard V. Effect of fibre debonding in a whisker-reinforced metal. Mater Sci Eng, Part A 1990;A125:203-13.

[32] Camacho G, Ortiz M. Computational modelling of impact damage in brittle materials. Int J Solids Struct 1996;33(20-22):2899-938.

[33] Geubelle P, Baylor J. Impact-induced delamination of composites: a 2d simulation. Composites: Part B 1998;29B:589-602.

[34] Campilho R, de Moura M, Domingues J. Modelling single and double-lap repairs on composite materials. Compos Sci Technol 2005;65:1948-58.

[35] Lebon F, Rizzoni R, Ronel S. Asymptotic analysis of a thin interface: the case involving similar rigidity. Int J Eng Sci 2010;48(5):473-86.

[36] Suquet P. Discontinuities and plasticity. In: Moreau, Panagiotopoulos, editors. Nonsmooth mechanics and applications. Wien: Springer-Verlag; 1988.

[37] Khaoua O, Lebon F, Licht C, Michaille G. Thin layer in elasticity: a theoretical and numerical study. Proceedings of third biennal European joint conference on engineering systems design and analysis. ASME 1996;4:165-70.

[38] Lebon F, Rizzoni R, Ronel S. Analysis of non-linear soft thin interfaces. Comput Struct 2004;82:1929-38.

[39] Chataigner S, Caron JF, Duong VA, Diaz AD. Experimental and numerical investigation of shear strain along an elasto-plastic bonded lap joint. Construct Build Mater 2011;25(2):432-41.

[40] Ladeveze P. Nonlinear computational structural mechanics - new approaches and non-incremental methods of calculation. Verlag: Springer; 1999.

[41] Gouri D, Touzot G. Une Présentation de la Méthode des Eléments Finis. S.A.: Maloine; 1984.

[42] Nguyen V. Modélisation globale et locale des structures multicouches par éléments finis de plaque. Ph.D. thesis, LAMI/ENPC; 2004. 